\title{
Black Box: Uma Aplicação de Gerenciamento e Entrega Tolerante a Atrasos e Desconexões de Vídeos Para Veículos
}

\author{
Charles T. B. Garrocho ${ }^{1}$, Maurício J. da Silva ${ }^{1}$, Ricardo A. R. Oliveira ${ }^{1}$ \\ ${ }^{1}$ Laboratório IMóbilis \\ Departamento de Computação - DECOM \\ Universidade Federal de Ouro Preto (UFOP) - Ouro Preto, MG - Brazil \\ \{ctgarrocho, badriciobq, rrabelo\}@gmail.com
}

\begin{abstract}
Develop, test and experience network applications for vehicles has become a major challenge for the scientific community, since most applications are evaluated in simulated form. Most of these applications are focused on the processing of information in real time. However, by mobility issues, it is virtually impossible distribute contents such as videos, because they are heavy, and thus unworkable in services such as mobile telephony, by resource constraints. To overcome these limitations, it's proposed a vehicular application which performs video recording, fragmenting them into smaller pieces and lower quality to be transmitted in encounters between the vehicles through an opportunistic network until they arrive at their destination, forming so a delay and disruption tolerant network. Experiments have shown that the application has successfully delivering video to the destination through the network created.
\end{abstract}

Resumo. Desenvolver, testar e experimentar aplicações de rede para veículos se tornou um grande desafio para a comunidade científica, uma vez que, a maioria das aplicações são avaliadas de forma simulada. A maioria dessas aplicações são focadas no processamento de informações em tempo real. Entretanto, por questões de mobilidade, é praticamente impossível distribuir conteúdos como vídeos, por serem pesados, e assim, inviáveis em serviços como telefonia móvel, por limitações de recursos. Para superar essas limitações, é proposto uma aplicação veicular que realiza o gerenciamento da gravação de vídeos, fragmentando-os em pedaços menores e com menor qualidade para serem transmitidos em encontros entre os veículos através de uma rede oportunista até que cheguem ao seu destino, formando assim uma rede tolerante a atrasos e desconexões. Experimentos demonstraram que a aplicação conseguiu com sucesso a entrega dos vídeos ao destino através da rede criada.

\section{Introdução}

Hoje em dia, as redes de comunicação sem fio tornaram-se essenciais no cotidiano de toda sociedade [Castells et al. 2009]. As pessoas podem se conectar a redes de dados, acessar e disponibilizar conteúdos em praticamente qualquer lugar e a qualquer momento, por meio de diferentes dispositivos e tecnologias de comunicação [Ma et al. 2014]. Isso se tornou possível principalmente graças ao avanço da rede celular (por exemplo, 3G (Third Generation of Mobile Telecommunications Technology), e LTE (Long Term Evolution)), com mais locais com acessos através de torres de transmissão e o aumento da capacidade de transmissão entre os dispositivos e as torres [Dahlman et al. 2013]. 
A pesar da evolução da rede celular, ela ainda não está disponível em muitos lugares e nem mesmo presente em grande parte da população de países com índice de desenvolvimento alto [Rainie 2010]. A rede celular também apresenta falhas de infraestrutura de comunicação, parciais ou totais causadas por desastres naturais [Skouloudi and Karsberg 2014], censura do governo [Deibert et al. 2011] ou por até uma interrupção de Internet [Chen 2011]. Além disso, as operadoras de telecomunicações tradicionais hesitam em investir em tais áreas por não gerarem lucro, uma vez que há riscos e custos elevados [Ims 2012].

A fim de superar essa falha e falta de conectividade para permitir as comunicações entre os dispositivos e aplicações em cenários perturbadores foi proposto as redes DTNs (Delay and Disruption Tolerant Networks) [Fall 2003]. As DTNs são um tipo de rede com conexão intermitente na qual um caminho fim-a-fim entre os nós que estão comunicando pode nunca existir [Fall and Farrell 2008]. Este tipo de abordagem de comunicação é feita através de oportunidades de encontros entre os nós que trocam dados entre si até que a mensagem chegue ao destinatário [Galati 2010].

As redes DTNs evoluirão muito nos últimos anos produzindo diversos protocolos e algoritmos de comunicação veicular [Vasilakos et al. 2011]. Esses trabalhos surgiram por que o veículo se tornou um lugar onde as pessoas ficam muito tempo durante seu dia-a-dia [Barfield and Dingus 2014], e assim, fornecer informação ao usuário no veículo se tornou um grande desafio, que tem sido explorado através dos SIT (Sistemas Inteligentes de Transporte) [Dimitrakopoulos and Demestichas 2010]. Entretanto, a maioria dos SIT são analisados através de simulações, e poucas avaliações reais através de aplicações práticas foram executadas em ambientes veiculares na literatura [Pereira et al. 2012]. Assim, desenvolver, testar e avaliar aplicações reais para veículos se tornou um desafio para a comunidade científica [Karagiannis et al. 2011].

Com o objetivo de preencher essa lacuna dos SIT, este trabalho tem como principal contribuição uma aplicação veicular, o Black Box, que realiza a gravação dos vídeos no veículo, fragmentando essa gravação em vários pedaços de vídeos com baixa qualidade com o objetivo de gerar arquivos de vídeos menores que possibilitem serem trocados entre veículos em curtos espaços de tempo. Essa troca de vídeos é realizada em encontros não programados entre os veículos através de uma arquitetura transparente [Garrocho et al. 2015] de rede oportunista.

Resultados de experimentos realizados na aplicação Black Box demonstraram que a arquitetura de rede oportunista utilizada se comporta melhor em locais onde há maior quantidade de veículos (por exemplo, vias congestionadas e sinais de trânsito). Além disso, os resultados demonstraram que a medida que a quantidade de veículos aumenta a taxa de transmissão diminui, e assim, a fragmentação dos vídeos é essencial para que a rede não seja sobrecarregada com a entrega dos vídeos.

O restante do artigo está organizado da seguinte forma: na Seção 2 são apresentados os trabalhos relacionados. Na Seção 3 é apresentado uma visão geral da aplicação Black Box. Na Seção 4 é feita uma avaliação do aplicação, sendo que na Subseção 4.1 são apresentados os cenários e métricas avaliadas, e na Subseção 4.2 são apresentados os resultados dos experimentos. Finalmente na Seção 5 são apresentadas as conclusões e os trabalhos futuros. 


\section{Trabalhos Relacionados}

Uma aplicação é uma coleção de instruções que descrevem uma tarefa a ser realizada por um dispositivo [Lloyd 2012]. Normalmente ela é desenvolvida para uma determinada tarefa gerando resultados esperados. Atualmente existem diversas aplicações que permitem a simulação de ambiente de redes DTN [Kaur and Malhotra 2015]. Essas simulações podem garantir um menor custo da avaliação de projetos e criar ambientes que são impossíveis por falta de tecnologia. Porém, os resultados de experimentos em ambientes reais continuam tendo maior impacto que ambientes simulados [Rutten et al. 2012].

Recentemente, em redes DTN, os protocolos de roteamentos foram mais abordados que aplicações práticas. Um grande número de algoritmos de encaminhamento, sem infraestruturas foram desenvolvidos. Os autores desses trabalhos baseiam-se que os dispositivos possuem a capacidade de troca e encaminhamento de mensagens através de uma rede Wireless Ad-Hoc, utilizando tecnologias sem fio [Wei et al. 2014]. Entretanto, existem na literatura algumas soluções de aplicações cuja meta é explorar as características inerentes as redes DTNs para a transmissão de mensagens, permitindo uma entrega de mensagens mesmo em falta de infraestrutura de comunicação [Vasilakos et al. 2011].

Um dos precursores de aplicações DTN foi o projeto ZebraNet [Juang et al. 2002]. Este sistema consiste em coleiras de rastreamento transportadas por zebras no âmbito de reservas da África. Os colares operam como forma de conexão oportunista para transmitir dados de volta para os pesquisadores. Isso significa que quando duas zebras se encontram, as coleiras registram as informações de encontro e trocam os dados armazenados em suas memórias locais. Através de oportunidades de encontro intermitentes, informação é gravada e depois difundida a essas zebras que transportam coleiras de rastreamento.

O sistema DakNet [Pentland et al. 2004] foi um projeto desenvolvido para criação de uma rede Ad-Hoc tolerante a atrasos e desconexões. O sistema DakNet consiste de quiosques com Wi-Fi habilitado em aldeias, e de dispositivos móveis que ao se aproximarem de quiosques de uma aldeia, detecta automaticamente as conexões sem fio e, em seguida, realiza a transmissão e recebimento de dados com os quiosques. Quando este dispositivo móvel entra no alcance de comunicação de um ponto de acesso à Internet, ele irá sincronizar automaticamente as mensagens dos quiosques das aldeias com à Internet.

Cartel [Hull et al. 2006] é um sistema de comunicação projetado para coletar, transmitir e visualizar dados a partir de sensores localizados em unidades móveis, como carros. Um nó CarTel (computador acoplado a um conjunto de sensores) coleta e processa a mensagem de sensores localizados em automóveis, e em seguida, entrega esses dados para um portal central (que funciona como ponto de controle e configuração para o sistema), utilizando CafNet. Em outras palavras, nós CarTel dependem de conectividade sem fio oportunista (por exemplo, Wi-Fi, Bluetooth) para se comunicar com um portal.

BikeNet [Eisenman et al. 2009] é um sistema de mapeamento das experiências de ciclistas. Ele usa uma série de sensores embarcados em uma bicicleta para coletar dados sobre os passeios de ciclistas. BikeNet encontra pontos de acesso sem fio de forma oportunista para troca de conteúdos, e aproveita o canal de dados celular dos ciclistas para comunicação em tempo real, se necessário. BikeNet também fornece um portal Web para o ciclista acessar seus dados, e permitir o compartilhamento desses dados (por exemplo, rotas de ciclismo favoritas, dados de poluição) com grupos interessados em ciclismo. 
Como pode ser observado, existem poucas aplicações práticas voltadas ao ambiente de redes DTN. O principal objetivo das aplicações é permitir uma entrega tolerante a atrasos e desconexões de um conteúdo á um destino. O sistema DakNet [Pentland et al. 2004] é o único que permite um acesso a internet sem ser em tempo real. Os trabalhos ZebraNet [Juang et al. 2002] e BikeNet [Eisenman et al. 2009] estão focados na difusão de informações sobre zebras e ciclistas. Já o sistema Cartel [Hull et al. 2006] esta voltado para o ambiente veicular, e é neste caso, o sistema mais parecido com o Black Box.

Cartel [Hull et al. 2006] está mais focado na distribuição de informações de tempo real dos veículos nas estradas, através de informações fornecidas pelos seus sensores. Essas informações podem ser utilizadas para evitar congestionamentos e acidentes nas estradas. Entretanto, nem todos os veículos possuem tais sensores. Já veículos pessoais, policiais e de transporte, em sua maioria já tem integrado câmeras que realizam filmagens tanto para dentro quanto para fora do veículo. A gravação de vídeo nesses veículos são utilizadas para monitoramento e detecção de movimentos de veículos em tempo real nas estradas [Jazayeri et al. 2011]. Porém os vídeos gravados por essas câmeras não só são utilizados no momento em que o veículo está em movimento, como também para uma análise posterior da conduta do motorista e dos passageiros, ou para análise de uma situação que tenha ocorrido no veículo ou próximo a ele [Noble and Alpert 2008]. Com o objetivo de auxiliar no gerenciamento de vídeos gravados nos veículos e na entrega posterior desses vídeos para análise, foi desenvolvido e avaliado o sistema Black Box.

\section{O Sistema Black Box}

Esta aplicação gerencia a gravação de vídeos em partes e realiza a entrega de vídeos solicitados do veículo para um servidor através de uma rede DTN formada através de uma arquitetura transparente [Garrocho et al. 2015] que fornece conectividade oportunista entre os veículos.

\section{Organização e Implementação}

A Figura 1 ilustra a organização dos componentes no veículo. Cada veículo é equipado de uma placa PandaBoardES ${ }^{1}$ e uma câmera que realiza o monitoramento do motorista.

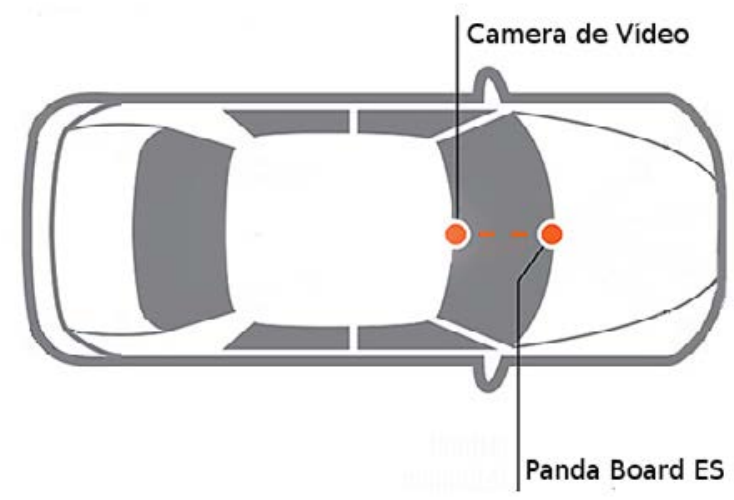

Figura 1. Organização dos componentes no veículo.

\footnotetext{
${ }^{1}$ http://pandaboard.org/content/pandaboard-es
} 
A aplicação Black Box foi desenvolvida sobre o SO (Sistema Operacional) Ubuntu 14.04, sendo utilizado uma versão totalmente customizada, que satisfaça apenas as necessidades da aplicação. A aplicação foi implementada para executar como um serviço para o SO, sendo assim, ele executa todas as suas funções em segundo plano.

\section{Funcionamento}

Quando a aplicação Black Box é executada na inicialização do SO móvel da placa PandaBoardES, é realizada uma operação de inicialização da arquitetura transparente de rede oportunista. Após isso, a aplicação executa diversas tarefas simultaneamente, realizando a gravação de vídeos da câmera instalada no veículo, transmissão dos vídeos através da rede oportunista, e gerenciamento do espaço disponível em disco apagando vídeos antigos.

A aplicação é divida em duas threads, sendo que a primeira thread realiza a gravação dos vídeos da câmera do veículo de tempo em tempo em uma pasta do sistema. O intervalo de tempo de gravação dos vídeos é definido em um arquivo de configuração, podendo assim, ser modificado a qualquer momento. O nome de identificação dos vídeos gravados na pasta do sistema é definido através do endereço MAC da placa juntamente com a data e hora atual do sistema, criando assim, uma identificação única.

A segunda thread realiza o gerenciamento do espaço disponível em disco do sistema, baseado em um limite de espaço definido em um arquivo de configuração, sendo possível sua modificação. Para realizar esse gerenciamento, a aplicação Black Box verifica que se caso o limite máximo exceda, o vídeo mais antigo é removido da pasta. Esse processo é executado de minuto em minuto através da ferramenta crontab $^{2}$, com o objetivo de sempre estar com um espaço disponível no sistema.

Quando o veículo está desligado ou em movimento, o sistema permanece ativo, e sistematicamente tenta trocar vídeos através de contatos oportunistas. Quando uma rede é estabelecida, e um vídeo é solicitado, conforme é ilustrado na Figura 2(a), Black Box automaticamente distribui o vídeo entre todos os veículos próximos.

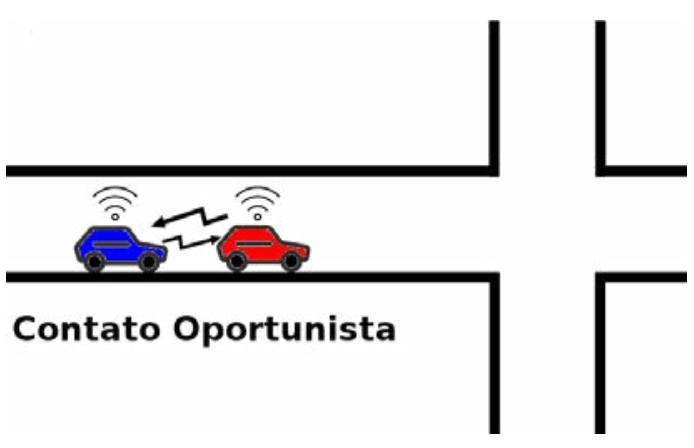

(a) Troca de vídeos entre os veículos.

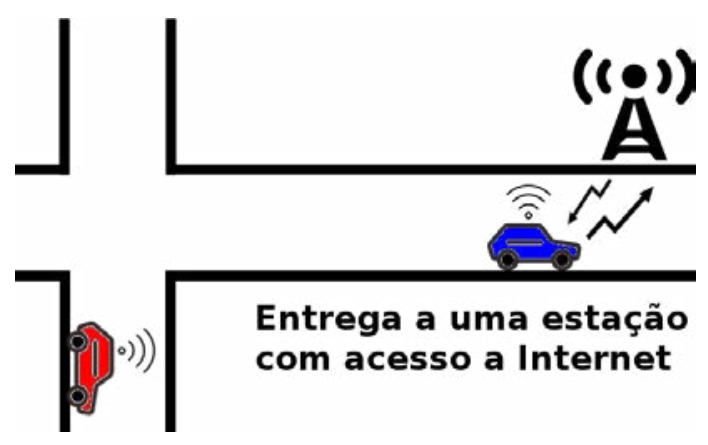

(b) Entrega de vídeos a uma infraestrutura.

Figura 2. Funcionamento da rede DTN criada pela aplicação Black Box.

Quando um veículo se encontra com uma infraestrutura instalada na via, conforme é ilustrado na Figura 2(b), a aplicação Black Box entrega todos os vídeos armazenados no dispositivo e que estão sendo solicitados para a infraestrutura. Assim, a infraestrutura, com conexão a internet, trata de entregar esses vídeos ao servidor destino.

\footnotetext{
${ }^{2}$ http://www.devin.com.br/crontab/
} 


\section{Avaliação do Black Box}

Nesta Seção são apresentados os cenários e métricas da aplicação Black Box para avaliação da rede DTN.

\section{Cenários e Métricas}

Em todos os experimentos que envolvem a transferência de arquivos no Black Box, os arquivos possuíam o tamanho de $1 \mathrm{MB}$. Esse tamanho representava o tamanho dos vídeos gravados nos veículos. Eles possuíam esse tamanho, por serem gravados em pequenas faixas de tempo, estarem em preto e branco, e terem uma resolução menor.

Foram realizados experimentos para avaliar o tempo de formação topológica de rede, a taxa de transmissão, e o tempo de entrega de arquivo tolerante a atrasos e desconexões. Os experimentos foram realizados em laboratório, de maneira controlada, não sendo considerado interferências e movimentos.

Os dados dos três experimentos foram obtidos realizando cálculos na própria aplicação Black Box, sendo considerado um intervalo de confiança de 95\%. Foi utilizado o padrão IEEE $802.11 \mathrm{~g}$, quadros de controle RTS/CTS e potência Tx padrão do dispositivo de $20 \mathrm{dBm}$.

No primeiro experimento, foi medido o tempo de associação topológica de rede entre os dispositivos. Cada dispositivo obtêm o tempo através do Log da aplicação Linux, que registra quando o dispositivo começa o processo de escaneamento de redes, e quando o dispositivo estabeleceu uma conexão. Cada dispositivo envia seu tempo ao dispositivo AP (Ponto de Acesso). Ao final o dispositivo AP faz cálculo com todos os tempo (inclusive o seu). Este experimento é importante para avaliar o impacto que a quantidade de dispositivos promove no tempo de formação da rede de comunicação.

No segundo experimento, a aplicação foi alterada de modo que 4 dispositivos requisitassem um arquivo de $1 \mathrm{MB}$ a um dispositivo. Foi medido a taxa de transmissão de pacotes entre os dispositivos através do cálculo dos tempos e do tamanho do arquivo. O principal objetivo deste experimento é avaliar como a rede de comunicação se comporta quando um dispositivo realiza a transmissão de um arquivo para vários dispositivos (quantidades diferentes) ao mesmo tempo.

No terceiro experimento, foi avaliado o tempo de entrega de um arquivo de $1 \mathrm{MB}$ em uma rede DTN. Cada operação (envio e recebimento) de arquivos juntamente com o tempo que a operação foi efetivada são armazenados em um Log para que posteriormente pudesse ser lidos e gerados as estatísticas de tempos de entrega dos arquivos. As estatísticas extraídas foram calculadas através da soma do tempo médio que cada arquivo (de cada dispositivo) teve para chegar em cada dispositivo participante da rede de comunicação, sendo considerado dois cenários, o primeiro de três dispositivos, e o segundo com quatro dispositivos.

Nos dois primeiros experimentos, os dispositivos ficaram sobre uma mesa com uma média de três metros de distância. Foram utilizadas 5 placas PandaBoardES, e todos os experimentos foram executados 30 vezes em cada dispositivo simultaneamente. Já no terceiro experimento as placas estavam separadas por um distância de 25 metros e utilizadas três a quatro, e os testes foram executados quatro vezes em cada dispositivo simultaneamente. 


\section{Resultados}

Nesta Seção são apresentados os resultados dos experimentos realizados no Black Box. A Figura 3 ilustra os resultados do primeiro experimento. Pode-se observar que, quando há poucos dispositivos, o tempo de formação de rede é grande, e sua faixa de erro também. Porém, quando a quantidade de dispositivos aumenta, o tempo de formação de rede diminui e a taxa de erro também. Isso acontece por que quando há poucos dispositivos, a probabilidade dos dispositivos ficarem ao mesmo tempo escaneando ou se tornando ponto de acesso é maior do que quando há uma quantidade maior de dispositivos.

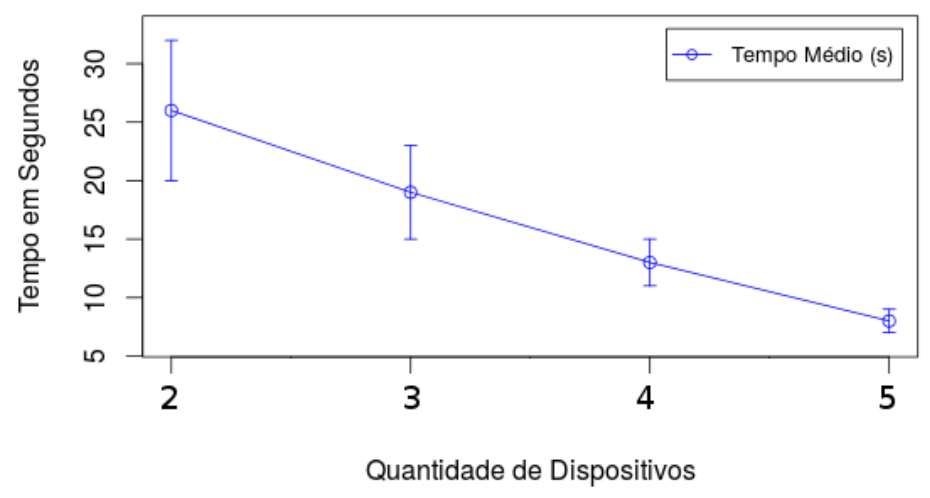

Figura 3. Tempo de formação da rede de comunicação.

A partir dos resultados do primeiro experimento, é possível concluir que o tempo de formação de rede, não é viável para comunicação com os veículos em movimento. Entretanto, esse sistema pode ser aplicado em locais onde os veículos se encontram e permanecem parados por alguns instantes de tempo (por exemplo, em um sinal de trânsito, ou em um estacionamento).

No segundo experimento, pode-se observar na Figura 4, que a quantidade de dispositivos influência na taxa de transmissão, onde a taxa diminui de acordo que a quantidade de dispositivos que recebem um arquivo aumenta. Isso acontece por que o dispositivo servidor que está cuidando de transmitir o arquivo, tem um trabalho maior com quantidade maiores de dispositivos, e assim, a banda de conexão desse dispositivo está mais ocupada com várias conexões simultâneas e mais pacotes para serem processados.

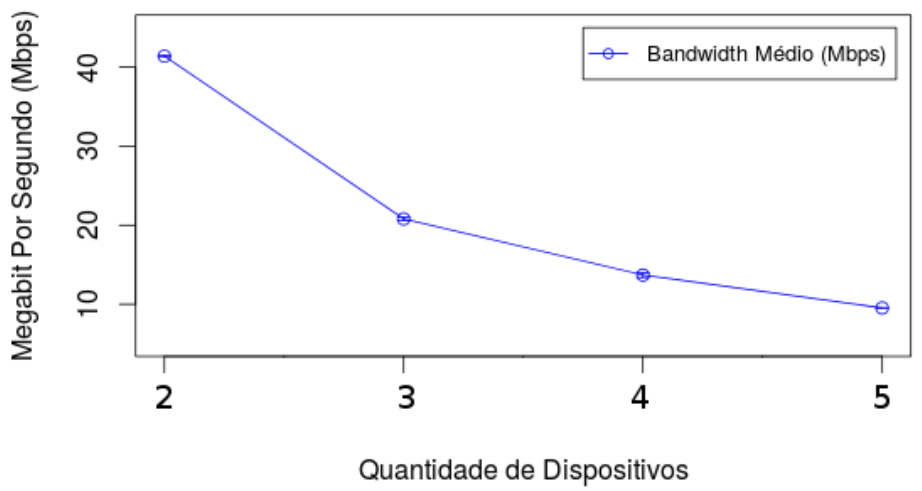

Figura 4. Taxa de transmissão de pacotes. 
A partir dos resultados apresentados na Figura 4, também é possível verificar que a taxa ficou menor que o limite do padrão $802.11 \mathrm{~g}$ e variou pouco. Isso aconteceu por causa da distância entre os dispositivos e o tamanho de arquivos utilizados em cada dispositivo. Variou menos por causa do tamanho do arquivo utilizado, no caso 1MB na aplicação. Já a taxa ficou menor por causa da distância entre os dispositivos, sendo de 15 metros.

No terceiro experimento, pode-se observar na Figura 5, que o que mais impactou no tempo de entrega de arquivos tolerante a atrasos e desconexões foi a distância entre os dispositivos. É possível visualizar que tanto para três quanto para quatro dispositivos, o tempo de entrega dos arquivos dos dispositivos que estavam nas extremidades é maior do que o tempo dos arquivos dos dispositivos que estavam no meio, servindo como ponto de comunicação entre os dispositivos das extremidades.

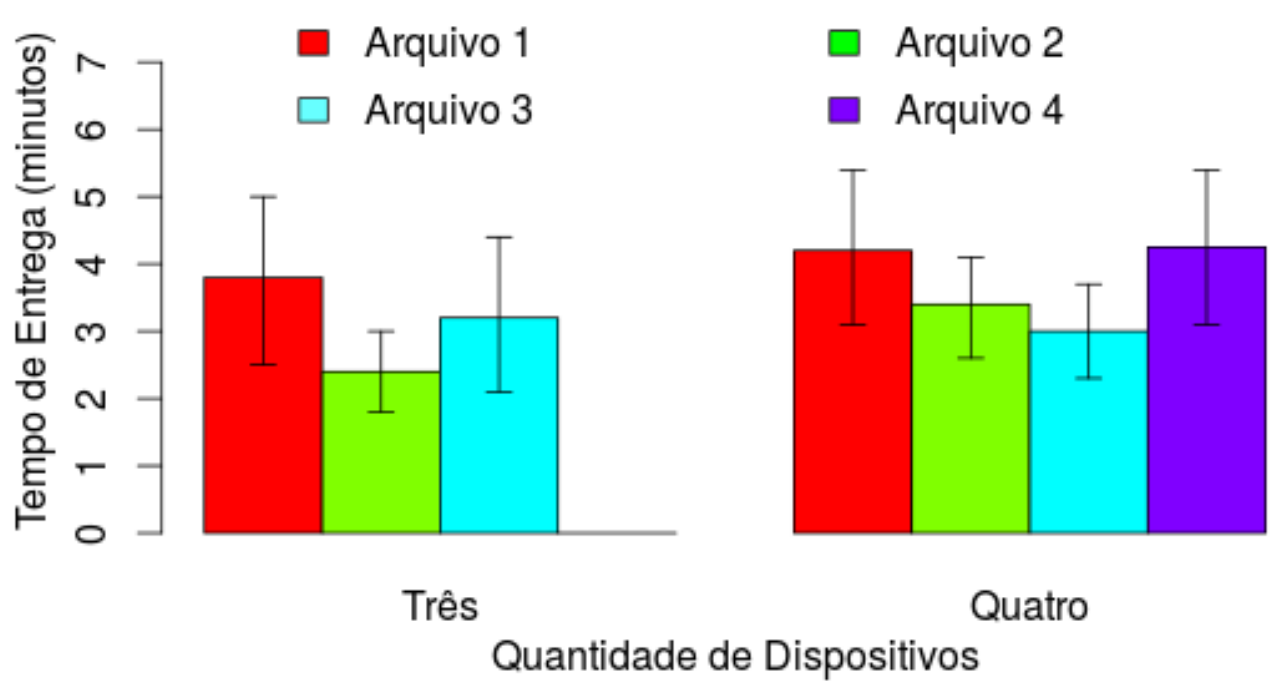

Figura 5. Tempo de entrega de arquivos em uma rede DTN.

A explicação dessa diferença de tempo deve-se principalmente ao fato de que os dispositivos que estão no meio se comunicam e entregam seus arquivos com um tempo menor aos dispositivos que estão próximos a eles, nesse caso, os dispositivos que estão na extremidade. O tempo de entrega dos arquivos dos dispositivos das extremidades ficou maior pelo fato de que para seus arquivos serem entregues de uma extremidade a outra, deve passar por um (três dispositivos) ou mais (quatro ou mais dispositivos) pontes de comunicação, e uma (três dispositivos) ou mais (quatro ou mais dispositivos) redes devem ser criadas para que isso aconteça.

Na Figura 5 também é possível visualizar uma taxa de erro grande no tempo de entrega de arquivos, principalmente nos tempos de entrega dos dispositivos das extremidades. Isso acontece por causa das redes formadas pelo middleware Net-Opp. Como o sistema baseia-se em tempos aleatórios para pesquisar e formar as redes, em alguns momentos, quando não há arquivos para serem transmitidos, o dispositivo não consegue encontrar um segundo dispositivo além do último que ele se comunicou, e ele acaba voltando a se comunicar com o primeiro, afetando assim, o tempo de entrega de seus arquivos e dos arquivos do primeiro dispositivo conectado à ele. 


\section{Conclusão e Trabalhos Futuros}

Neste artigo, foi apresentado o Black Box, uma aplicação que realiza o gerenciamento eficiente de vídeos em veículos e permite a entrega de vídeos solicitados a um servidor através de uma rede DTN criada pela aplicação.

Os resultados apresentados neste artigo, mostraram em experiências reais a eficiência de Black Box na formação de rede e na troca de conteúdos entre os veículos, sendo escalável e promovendo uma comunicação transparente. Mostrando-se viável sua aplicação em locais onde os veículos se encontram e permanecem parados por alguns instantes de tempo (por exemplo, em um sinal de trânsito, ou em um estacionamento).

Como trabalhos futuros, além do aprimoramento da aplicação Black Box, pretende-se estender os estudos, empregando o uso da aplicação em diferentes cenários e maiores quantidades de dispositivos, com o propósito de avaliar mais profundamente o comportamento da aplicação nessa estrutura de rede.

\section{Referências}

Barfield, W. and Dingus, T. A. (2014). Human factors in intelligent transportation systems. Psychology Press.

Castells, M., Fernandez-Ardevol, M., Qiu, J. L., and Sey, A. (2009). Mobile communication and society: A global perspective. Mit Press.

Chen, T. M. (2011). Governments and the executive"internet kill switch"[editor's note]. Network, IEEE, 25(2):2-3.

Dahlman, E., Parkvall, S., and Skold, J. (2013). 4G: LTE/LTE-advanced for mobile broadband. Academic press.

Deibert, R., Palfrey, J., Rohozinski, R., and Zittrain, J. (2011). Access contested: security, identity, and resistance in Asian cyberspace. MIT Press.

Dimitrakopoulos, G. and Demestichas, P. (2010). Intelligent transportation systems. Vehicular Technology Magazine, IEEE, 5(1):77-84.

Eisenman, S. B., Miluzzo, E., Lane, N. D., Peterson, R. A., Ahn, G.-S., and Campbell, A. T. (2009). Bikenet: A mobile sensing system for cyclist experience mapping. ACM Transactions on Sensor Networks (TOSN), 6(1):6.

Fall, K. (2003). A delay-tolerant network architecture for challenged internets. In Proceedings of the 2003 conference on Applications, technologies, architectures, and protocols for computer communications, pages 27-34. ACM.

Fall, K. and Farrell, S. (2008). Dtn: an architectural retrospective. Selected Areas in Communications, IEEE Journal on, 26(5):828-836.

Galati, A. (2010). Delay tolerant network. Germany: LAP Lambert Academic Publishing.

Garrocho, C. T. B., Silva, M. J., and Oliveira, R. A. R. (2015). Transparent sharing architecture of content between mobile devices in opportunistic networks. In Proceedings of the annual conference on Brazilian Symposium on Information Systems: Information Systems: A Computer Socio-Technical Perspective-Volume 1, page 48. Brazilian Computer Society. 
Hull, B., Bychkovsky, V., Zhang, Y., Chen, K., Goraczko, M., Miu, A., Shih, E., Balakrishnan, H., and Madden, S. (2006). Cartel: a distributed mobile sensor computing system. In Proceedings of the 4th international conference on Embedded networked sensor systems, pages 125-138. ACM.

Ims, L. A. (2012). Broadband access networks: introduction strategies and technoeconomic evaluation. Springer Science \& Business Media.

Jazayeri, A., Cai, H., Zheng, J. Y., and Tuceryan, M. (2011). Vehicle detection and tracking in car video based on motion model. Intelligent Transportation Systems, IEEE Transactions on, 12(2):583-595.

Juang, P., Oki, H., Wang, Y., Martonosi, M., Peh, L. S., and Rubenstein, D. (2002). Energy-efficient computing for wildlife tracking: Design tradeoffs and early experiences with zebranet. In ACM Sigplan Notices, volume 37, pages 96-107. ACM.

Karagiannis, G., Altintas, O., Ekici, E., Heijenk, G., Jarupan, B., Lin, K., and Weil, T. (2011). Vehicular networking: A survey and tutorial on requirements, architectures, challenges, standards and solutions. Communications Surveys \& Tutorials, IEEE, 13(4):584-616.

Kaur, A. and Malhotra, J. (2015). A survey of network simulation tools. Wireless Communication, 7(6):191-194.

Lloyd, J. W. (2012). Foundations of logic programming. Springer Science \& Business Media.

Ma, H., Zhao, D., and Yuan, P. (2014). Opportunities in mobile crowd sensing. Communications Magazine, IEEE, 52(8):29-35.

Noble, J. J. and Alpert, G. P. (2008). Managing accountability systems for police conduct: Internal affairs and external oversight. Waveland Press.

Pentland, A., Fletcher, R., and Hasson, A. (2004). Daknet: Rethinking connectivity in developing nations. Computer, 37(1):78-83.

Pereira, P. R., Casaca, A., Rodrigues, J. J., Soares, V. N., Triay, J., and Cervelló-Pastor, C. (2012). From delay-tolerant networks to vehicular delay-tolerant networks. Communications Surveys \& Tutorials, IEEE, 14(4):1166-1182.

Rainie, L. (2010). Internet, broadband, and cell phone statistics. Pew Internet \& American Life Project, 5.

Rutten, N., van Joolingen, W. R., and van der Veen, J. T. (2012). The learning effects of computer simulations in science education. Computers \& Education, 58(1):136-153.

Skouloudi, C. and Karsberg, C. (2014). Annual incident reports 2014. European Union Agency for Network and Information Security (ENISA).

Vasilakos, A. V., Zhang, Y., and Spyropoulos, T. (2011). Delay tolerant networks: Protocols and applications. CRC press.

Wei, K., Liang, X., and Xu, K. (2014). A survey of social-aware routing protocols in delay tolerant networks: applications, taxonomy and design-related issues. Communications Surveys \& Tutorials, IEEE, 16(1):556-578. 